\title{
Diagnóstico de necesidades de formación de los docentes de bachillerato y su pertinencia en la enseñanza - aprendizaje en los estudiantes previo a la prueba Ser Bachiller. Unidad Educativa Internacional Ibero Americano, Cantón Riobamba, provincia de Chimborazo.
}

Diagnosis of training needs of high school teachers and their relevance in teaching - learning in students prior to the Baccalaureate test. IberoAmerican International Educational Unit, Cantón Riobamba, province of Chimborazo.

Alex Fabián Inca Falconí. ${ }^{1}$, Maria Elizabeth Barahona Avecillas. ${ }^{2}$, Rosa Ximena Castelo Gavilanes. ${ }^{3} \&$ Jonatahn Paúl Campos Castelo. ${ }^{4}$

\begin{abstract}
.
DOI: $\underline{\text { https://doi.org/10.33262/concienciadigital.v4i3.2.1871 }}$

Introduction. At present, in a globalized world with constant technological development, changes are being promoted in different fields such as health, companies, the media, among others. Objective. The present research work entitled Diagnosis of training needs of high school teachers and their relevance in teaching-learning in students prior to the Baccalaureate test; It was carried out with the objective of knowing the training needs of

\footnotetext{
${ }^{1}$ Escuela Superior Politécnica de Chimborazo, Riobamba, Ecuador. alex.inca@espoch.edu.ec, ORCID: https://orcid.org/0000-0002-3312-6692

2 Instituto Superior Tecnológico "Carlos Cisneros", Riobamba, Ecuador. mabeth_baraa@hotmail.com. ORCID: https://orcid.org/0000-0001-9773-709X

3 Instituto Superior Tecnológico "Carlos Cisneros", Riobamba, Ecuador. ximcast@yahoo.es. ORCID: https://orcid.org/0000-0002-9993-2769

${ }^{4}$ Instituto Superior Tecnológico "Carlos Cisneros", Riobamba, Ecuador.jpcc_555@ hotmail.com. ORCID: https://orcid.org/0000-0002-8243-451X
} 
the high school teachers of the Ibero-American International Educational Unit of the Riobamba canton, Chimborazo province, in the academic period 2016 - 2017. Methodology. For the research process, the entire population was taken as a sample, made up of nine teachers and seventy high school students; the analytical-synthetic method, the inductive and deductive method, the statistical method, the hermeneutical method was put into practice; With the survey as a technique and the questionnaire as a tool, data was collected, which were classified and analyzed through statistical tables. Results. It was possible to detect that $90 \%$ of high school teachers have training needs in topics related to pedagogy, didactics, methodological strategies, activities for the development of skills, preparation and evaluation of projects, neurophysiology of learning. Conclusion. According to the analysis carried out, the high school teachers have third level academic training; there are teachers who have not been trained in the field of pedagogy.

Keywords: research, needs, training, teaching, learning

\section{Resumen.}

Introducción. En la actualidad ante un mundo globalizado y de constante desarrollo tecnológico se están promoviendo cambios en los diferentes campos como la salud, las empresas, medios de comunicación, entre otros. Objetivo. El presente trabajo de investigación titulado Diagnóstico de necesidades de formación de los docentes de bachillerato y su pertinencia en la enseñanza-aprendizaje en los estudiantes previo a la prueba Ser Bachiller; se realizó con el objetivo de conocer las necesidades de formación de los docentes de bachillerato de la unidad Educativa Internacional Ibero Americano del cantón Riobamba, provincia de Chimborazo, en el periodo académico 2016 - 2017. Metodología. Para el proceso de investigación se tomó como muestra la totalidad de la población, conformada por nueve docentes y setenta estudiantes de bachillerato; se puso en práctica el método analítico-sintético, el método inductivo y el deductivo, el método estadístico, el método hermenéutico; con la encuesta como técnica y como herramienta el cuestionario, se realizó la recolección de datos los mismos que fueron clasificados y analizados a través de tablas estadísticas. Resultados. Se logró detectar que un $90 \%$ de los docentes de bachillerato tienen necesidades de formación en temas referentes a pedagogía, didáctica, estrategias metodológicas, actividades para el desarrollo de destrezas, preparación y evaluación de proyectos, neurofisiología del aprendizaje. Conclusión. De acuerdo al análisis realizado los docentes de bachillerato tienen formación académica de tercer nivel; existiendo docentes que no se han formado en el ámbito de la pedagogía.

Palabras claves: investigación, necesidades, formación, enseñanza, aprendizaje. 


\section{Introducción.}

A través de los siglos el ser humano ha creado un conjunto de herramientas y máquinas de En la actualidad ante un mundo globalizado y de constante desarrollo tecnológico se están promoviendo cambios en los diferentes campos como la salud, las empresas, medios de comunicación, entre otros. Por lo expuesto anteriormente y considerando a la educación como el principal medio de formación de los futuros ciudadanos, quienes deben ser preparados para enfrentar las diferentes situaciones de su entorno este campo requiere de la mayor atención a las diferentes necesidades que se generan a través de sus actores. Es así que en nuestro país el campo de la educación se encuentra experimentando cambios muy importantes; tales como reformas a la LOEI y su Reglamento; el Sistema Nacional de Educación actualmente considera los siguientes niveles de educación: educación inicial, educación general básica, bachillerato. Los estudiantes que concluyen su formación de bachillerato deben rendir un examen de evaluación nacional conocida como la prueba "Ser Bachiller" para obtener el aval respectivo como bachiller de la república del Ecuador. Los docentes de nuestro país ven la necesidad de prepararse continuamente para cumplir con los objetivos propuestos por el Ministerio de educación, el mismo que dispone de diferentes programas de formación continua y capacitación. Por diferentes situaciones no todos los docentes se encuentran en procesos de formación continua o capacitación.

Existen investigaciones relacionadas que se tomaron como guía para la presente investigación; el estudio de (Calva, 2014) realizado en el Colegio Técnico Agropecuario Zumbi, analiza las necesidades de formación de los docentes de bachillerato, al finalizar su trabajo detecta que el mayor porcentaje de necesidades de formación son en pedagogía educativa, métodos y recursos didácticos.

(Robles, 2014) su trabajo sobre las necesidades de formación de los docentes de bachillerato de la Unidad Educativa Fiscomisional Pacífico Cembranos se caracteriza por su amplia investigación bibliográfica de las teorías y estudios referentes a necesidades formativas de los docentes. Llegando al final de su trabajo a la conclusión que existe la necesidad de planificar cursos de elaboración de proyectos educativos y de aula con la inserción de las TIC.

(Poveda, 2014) en su trabajo de investigación sobre las necesidades de formación de los docentes de bachillerato del colegio Gran Colombia, manifiesta que el análisis de los resultados demostró que existen necesidades de formación tanto en aspectos: disciplinares, pedagógicos, administrativos y psicológicos. En su conclusión general estima que los docentes de la Institución tienen necesidades formativas que satisfacer para cumplir satisfactoriamente con los requerimientos de la LOEI.

Frente a los cambios en el sistema de educación en nuestro país; las autoridades de la Unidad Educativa Internacional Ibero Americano, consideraron la importancia del aporte que puede dar un estudio sobre las necesidades de formación de los docentes de bachillerato, que permita establecer si existe tal demanda. Por tal razón con el presente trabajo se propuso la realización de un diagnóstico de la situación real de la formación de 
los docentes y su pertinencia en la enseñanza- aprendizaje en los estudiantes previo a la prueba Ser Bachiller.

La predisposición de las autoridades de la Unidad, la colaboración de los docentes y de los estudiantes permitieron la realización del trabajo; después de haber cumplido con el proceso de investigación, se pudo detectar que si existe la demanda de necesidades de formación docente que permita conocer muchos aspectos sobre los cambios del sistema, capacitarse sobre temas que el docente considera de mucha importancia para desarrollar sus actividades acordes a las demandas del sistema.

\section{Metodologia.}

Diseño Plan o estrategia que se desarrolla para obtener la información que se requiere en una investigación. (Hernandéz, 2010).

Los métodos de investigación que se aplicará a este estudio son el descriptivo, analítico y sistemático, que permite explicar y analizar el objeto de exploración.

Para facilitar la explicación y caracterización de la gestión en el liderazgo educativo y la promoción de valores en un centro educativo, el tipo de investigación a realizar es exploratorio y descriptivo tal cual se presenta en la realidad.

\section{Métodos de investigación.}

La lectura, es el medio importante para conocer, analizar y seleccionar aportes teóricos, conceptuales y metodológicos sobre la investigación a realizar.

\section{Técnicas de investigación.}

Son procedimientos metodológicos y sistemáticos que se encargan de recoger información de manera inmediata, las técnicas son también una invención del hombre y como tal existen tantas técnicas como problemas susceptibles de ser investigados.

Dependiendo del tipo de investigación que se realiza las técnicas de recolección de información están suscritas a dos formas muy conocidas: La primera a aquella que puede utilizar la información existente denominada información secundaria y la segunda que trabaja con información existente acerca de determinado tema llamada información primaria.

La encuesta: tiene la ventaja de formular preguntas a más personas quienes proporcionan. Información de sus condiciones económicas, familiares, sociales, culturales y políticas y en los que el anonimato constituye una ventaja porque no puede personalizarse. (Centty, 2010)

La observación este método de recolección de datos consiste en el registro sistemático, válido y confiable de comportamientos y situaciones observadas, a través de un conjunto de categorías y subcategorías. (Hernandéz, 2010) 


\section{Instrumentos de investigación.}

Cada día es más común ver estudios donde se utilizan diferentes métodos de recolección de datos. En los estudios cuantitativos no resultas extraño que se incluyan varios tipos de cuestionarios al mismo tiempo que pruebas estandarizadas y recopilación de contenidos para análisis estadístico u observación. (Hernandéz, 2010).

El cuestionario es un instrumento de mayor utilidad para recolectar; consiste en un conjunto de preguntas respecto de una o más variables a medir.

Preguntas.

- ¿Cuál es la pertinencia en la enseñanza - aprendizaje en los estudiantes de bachillerato previo al examen Ser Bachiller?

- ¿Qué tipo de necesidades de formación docente existe en los profesores de bachillerato?

- ¿Cuál es la reacción entre las dos variables investigadas?

\section{Población y Muestra.}

Población o universo conjunto de todos los casos que concuerdan con determinadas especificaciones. (Hernández, 2010).

El estudio se realizó en el total de la población: 9 docentes y 70 estudiantes de bachillerato.

\section{Gráfico 1}

Cuestionario aplicado a docentes de bachillerato del Liceo Internacional Iberoamericano.

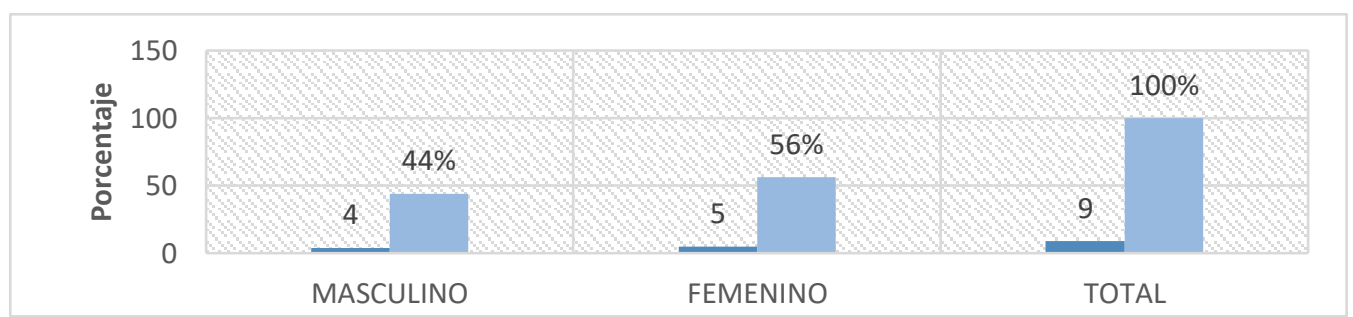

Fuente: Cuestionario aplicado a los estudiantes de bachillerato del Liceo Internacional Iberoamericano.

El personal docente de bachillerato de la Unidad educativa; está conformado por el $44 \%$ del género masculino, el $56 \%$ del género femenino. Cox en su modelo para el análisis de necesidades formativas considera que uno de los aspectos que se debe conocer son las características de las personas involucradas en la problemática; También se ha considerado los cuatro elementos del modelo Rosett para el análisis de las necesidades de formación docente. En base a los resultados obtenidos podemos determinar que el mayor porcentaje del personal docente es de género femenino. 


\section{Grafico 2}

Cuestionario aplicado a docentes de bachillerato del Liceo Internacional Iberoamericano

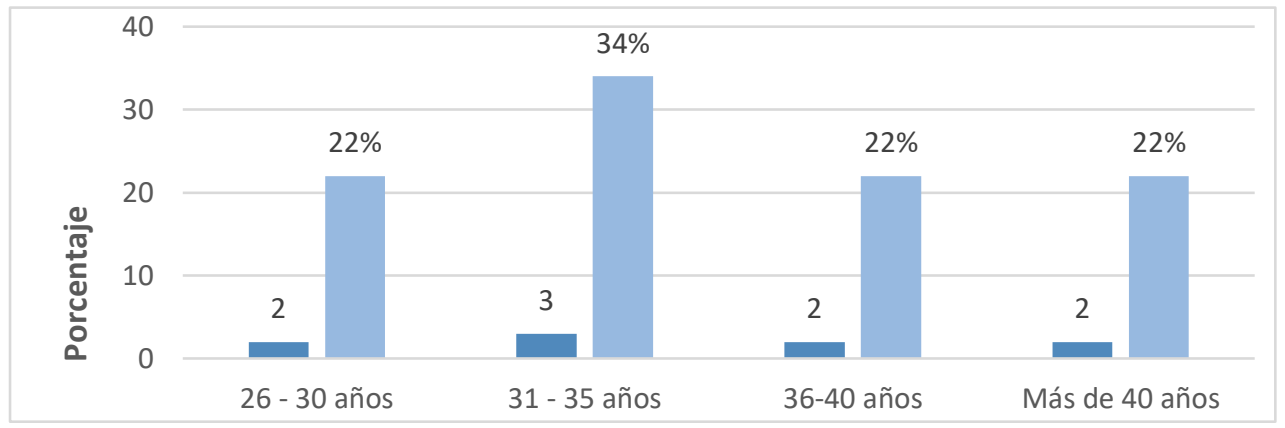

Fuente: Cuestionario aplicado a los estudiantes de bachillerato del Liceo Internacional Iberoamericano.

\section{Gráfico 3}

Cuestionario aplicado a docentes de bachillerato del Liceo Internacional Iberoamericano

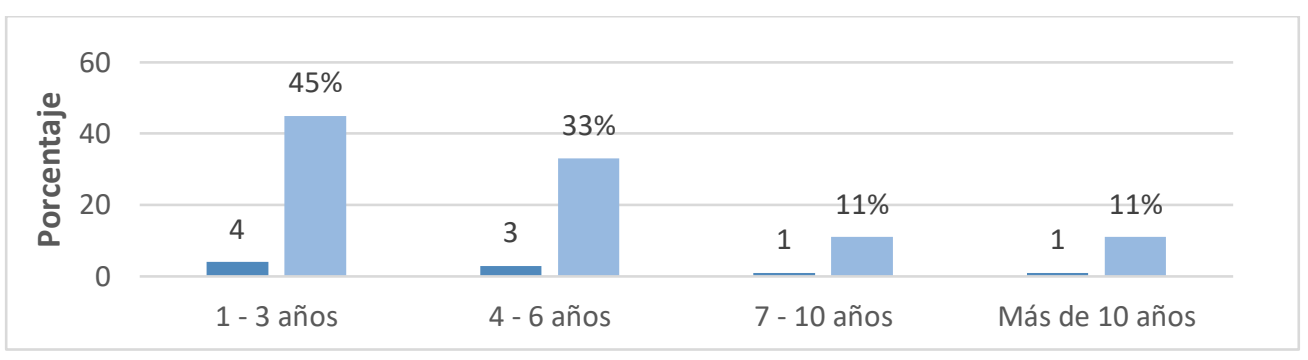

Fuente: Cuestionario aplicado a los estudiantes de bachillerato del Liceo Internacional Iberoamericano.

\section{Gráfico 4}

Cuestionario aplicado a docentes de bachillerato del Liceo Internacional Iberoamericano

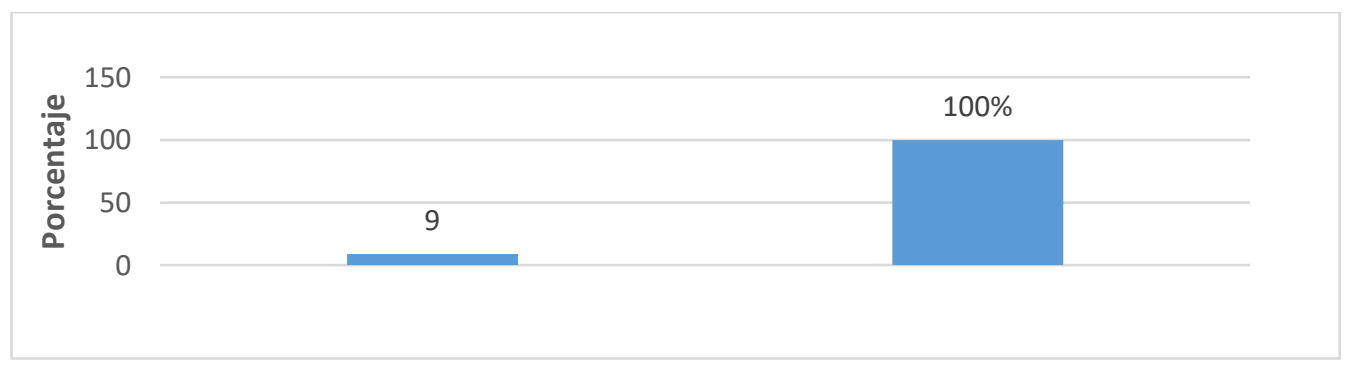

Fuente: Cuestionario aplicado a los estudiantes de bachillerato del Liceo Internacional Iberoamericano.

El 100\% de los docentes de bachillerato tienen tercer nivel de formación académica. Es una de las características muy importante sobre los involucrados en el problema para nuestro estudio considerando lo expuesto por Cox y como dato indispensable para nuestro análisis de acuerdo con el criterio del modelo de Rosett con el propósito de una adecuada detección de necesidades de formación docente. 


\section{Gráfico 5}

Cuestionario aplicado a docentes de bachillerato del Liceo Internacional

Iberoamericano

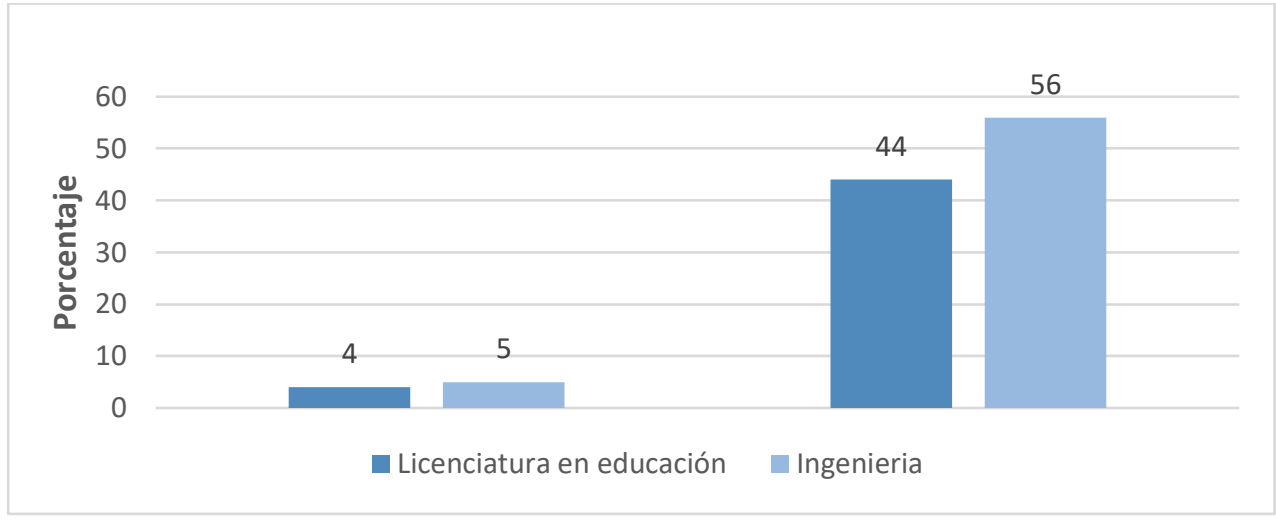

Fuente: Cuestionario aplicado a los estudiantes de bachillerato del Liceo Internacional Iberoamericano.

Los datos de la encuesta nos muestran que 4 docentes tienen título en licenciatura en educación lo que implica que tienen conocimientos de pedagogía, y cinco docentes tienen formación académica en ingeniería. Una característica muy importante que permitirá determinar la existencia de necesidades de formación docente tomando el criterio de los aspectos considerados en el modelo de Cox; y en base a los cuatro elementos del modelo de Rosett se podrá conocer la situación inicial de los involucrados y de apoyo en la detección de necesidades de formación que podrían tener los docentes de bachillerato.

\section{Gráfico 6}

Cuestionario aplicado a docentes de bachillerato del Liceo Internacional Iberoamericano

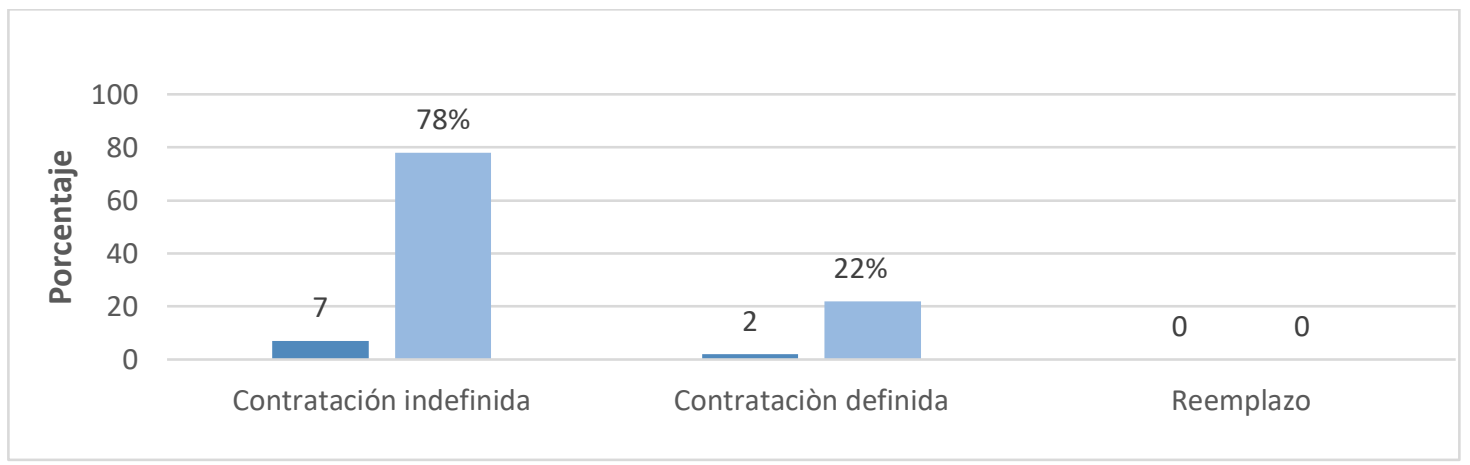

Fuente: Cuestionario aplicado a los estudiantes de bachillerato del Liceo Internacional Iberoamericano.

Referente a la relación laboral la tabla nos muestra que $78 \%$ de los docentes de bachillerato tienen contratación indefinida y el $22 \%$ tiene contratación definida. Forma parte de la investigación el estudio de esta condición por considerarla importante para el análisis de la situación inicial; como contempla el modelo de Rosett. La situación de los docentes de bachillerato en un alto porcentaje tiene contratación indefinida. 


\section{Gráfico 7}

Cuestionario aplicado a docentes de bachillerato del Liceo Internacional

Iberoamericano

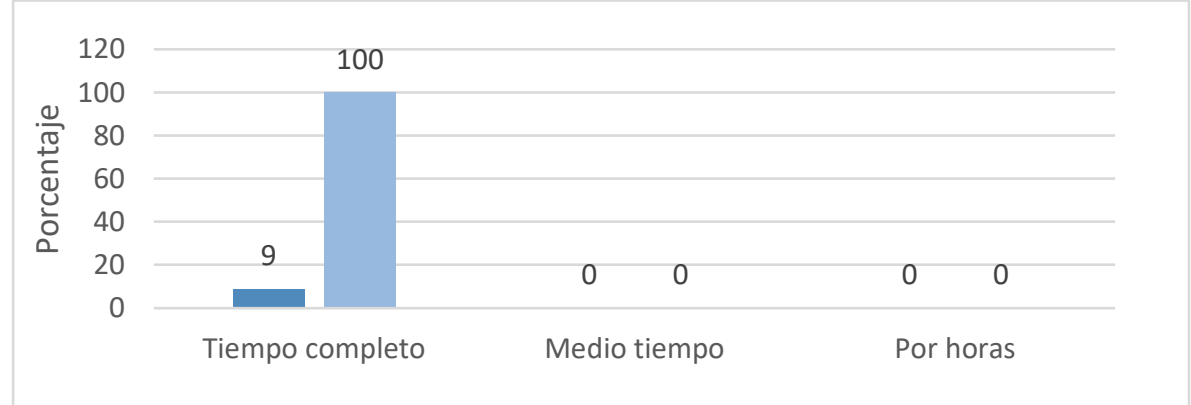

Fuente: Cuestionario aplicado a los estudiantes de bachillerato del Liceo Internacional Iberoamericano.

El resultado presentado en la tabla nos muestra que los 9 docentes de bachillerato es decir $100 \%$ trabajan a tiempo completo en la unidad educativa. Otra característica de las personas implicadas en el problema, tomado de los aspectos del método de Cox. Y se ha considerado los cuatro elementos del método de Rosett para conocer la situación inicial de los docentes. La situación inicial de los docentes de bachillerato en base a los resultados podemos establecer que trabajan a tiempo completo en la institución.

\section{Gráfico 8}

Cuestionario aplicado a docentes de bachillerato del Liceo Internacional Iberoamericano

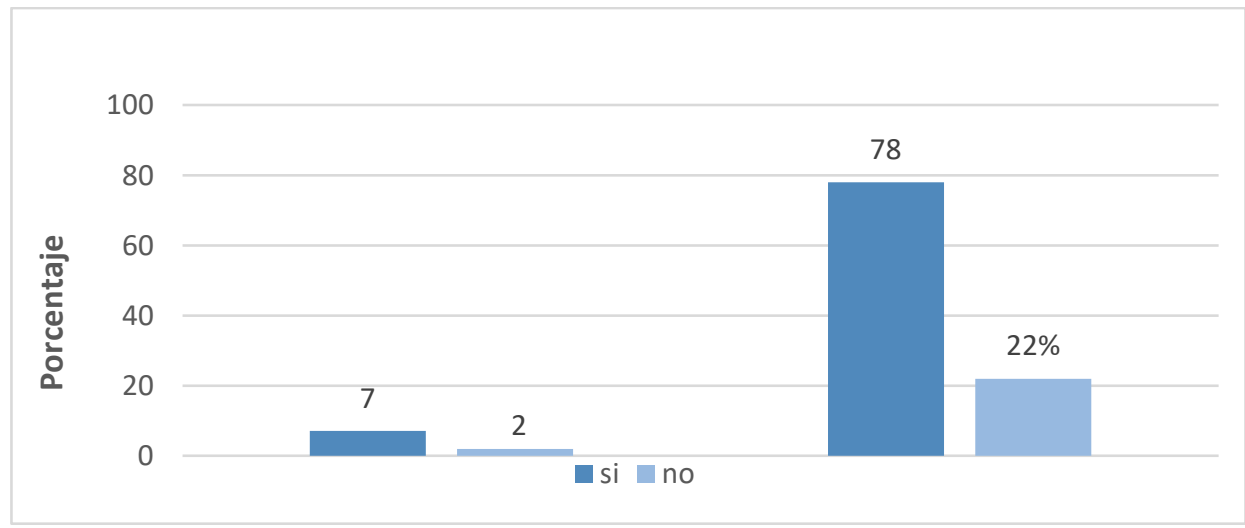

Fuente: Cuestionario aplicado a los estudiantes de bachillerato del Liceo Internacional Iberoamericano.

Los datos obtenidos de la encuesta; refleja que 7 docentes que equivale al $78 \%$ de los encuestados, se encuentran impartiendo materias que tienen relación con su formación académica; y 2 docentes correspondiente al $22 \%$ de los encuestados no se encuentran impartiendo materias en relación a su formación académica. Conocer las características y la situación inicial en la que se encuentran los involucrados en el problema se tomó en consideración para el estudio de los modelos de Cox y Rosett, Como resultado final se establece que existe un bajo porcentaje de docentes que se encuentran en el caso de impartir materias no relacionadas con su formación académica. 


\section{Formación docente.}

\section{Gráfico 9}

Cuestionario aplicado a docentes de bachillerato del Liceo Internacional Iberoamericano

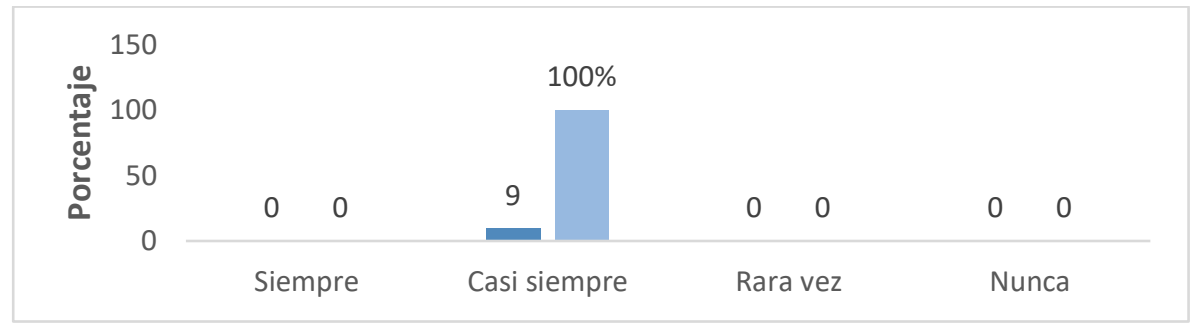

Fuente: Cuestionario aplicado a los estudiantes de bachillerato del Liceo Internacional Iberoamericano.

El 100\% de los encuestados opinan que casi siempre es coherente la formación académica y la gestión docente con las nuevas reformas estratégicas del sistema educativo. MinEduc dice: La respuesta generalizada de los docentes de bachillerato, denota la posibilidad de alguna falencia en el proceso de enseñanza que no permite alcanzar en su totalidad los objetivos propuestos por el sistema de educación.

\section{Gráfico 10}

Cuestionario aplicado a docentes de bachillerato del Liceo Internacional Iberoamericano

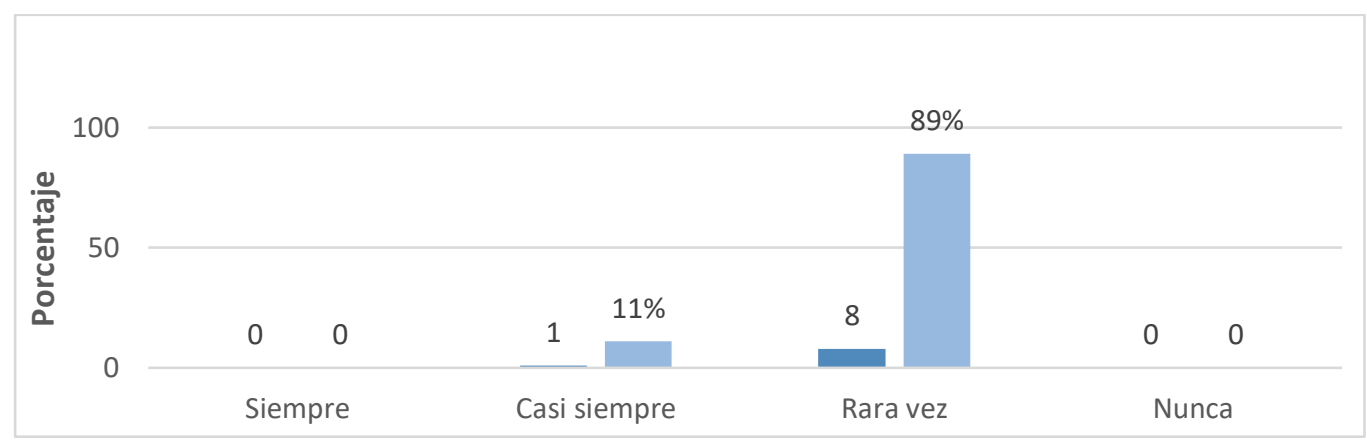

Fuente: Cuestionario aplicado a los estudiantes de bachillerato del Liceo Internacional Iberoamericano.

Hay 8 docentes correspondiente al $89 \%$ de los encuestados, manifiestan que rara vez participan en cursos de formación continua, y 1 docente es el único que participa en cursos de formación continua-

En consideración al nuevo esquema de aprendizaje del nuevo bachiller que el Mineduc establece; la formación continua es de vital importancia en un proceso de transición, el docente debe mantenerse en constante actualización por una parte para alcanzar los objetivos propuestos por el sistema, y por otra el beneficio debe enfocarse para el estudiante. El resultado final nos permite establecer que los docentes de bachillerato de la institución, requieren de formación continua para que su trabajo en la enseñanza sea coherente con los nuevos lineamientos del sistema. 


\section{Gráfico 11}

Cuestionario aplicado a docentes de bachillerato del Liceo Internacional Iberoamericano.

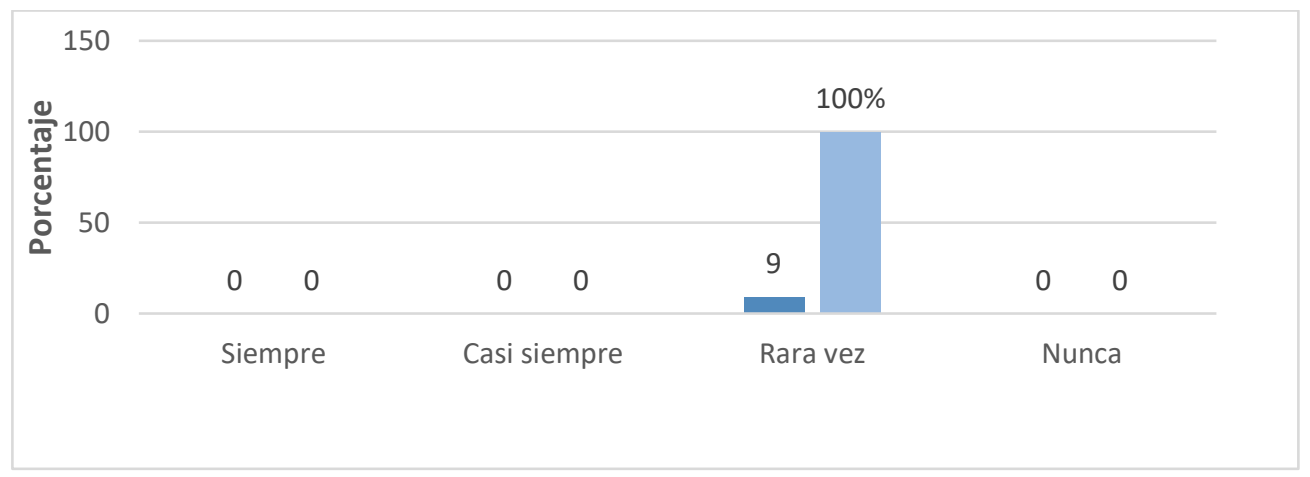

Fuente: Cuestionario aplicado a los estudiantes de bachillerato del Liceo Internacional Iberoamericano.

Los datos presentados en la tabla reflejan que 9 docentes el 100\% de los encuestados, manifiestan que rara vez la institución convoca a los docentes de bachillerato a capacitaciones. Considerando lo expuesto por (Daza, 2010) “(...) La importancia de reconocer una institución como un todo imprime a su comunidad el mensaje de que ese es el fin y que todas las unidades académicas y administrativas deben trabajar mancomunadamente para conseguirlo". Si la institución "rara vez" convoca a capacitaciones, procesos de formación y actualización; sus autoridades deben considerar en la planificación temas de formación docente.

\section{Gráfico 12}

Cuestionario aplicado a docentes de bachillerato del Liceo Internacional Iberoamericano

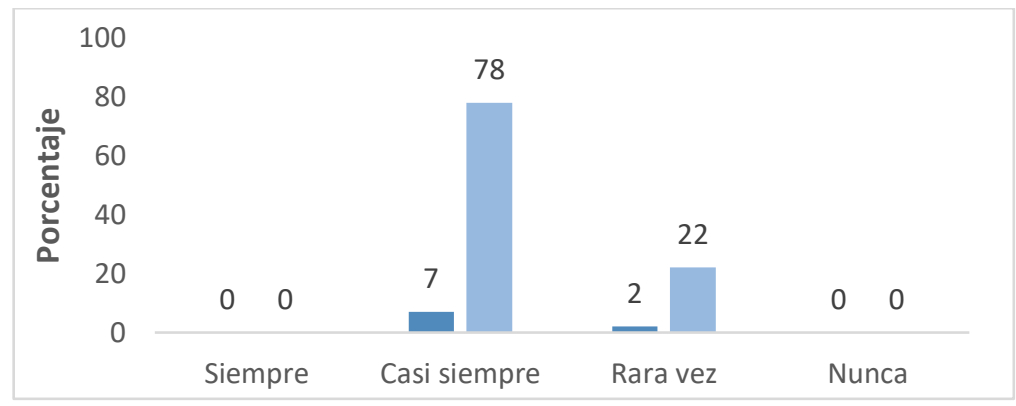

Fuente: Cuestionario aplicado a los estudiantes de bachillerato del Liceo Internacional Iberoamericano.

Los datos nos indican que el $78 \%$ de los docentes, manifiestan la aplican nuevas formas de pedagogía, el 22\% indican que rara vez. Acogiendo la experiencia compartida por Bedoya José (2005) y los resultados obtenidos. Nos permite concretar, que los docentes de la institución van desarrollando sus actividades de educación enmarcados en los nuevos retos que se le presentan día a día en su actividad. 


\section{Gráfico 13}

Cuestionario aplicado a docentes de bachillerato del Liceo Internacional

Iberoamericano

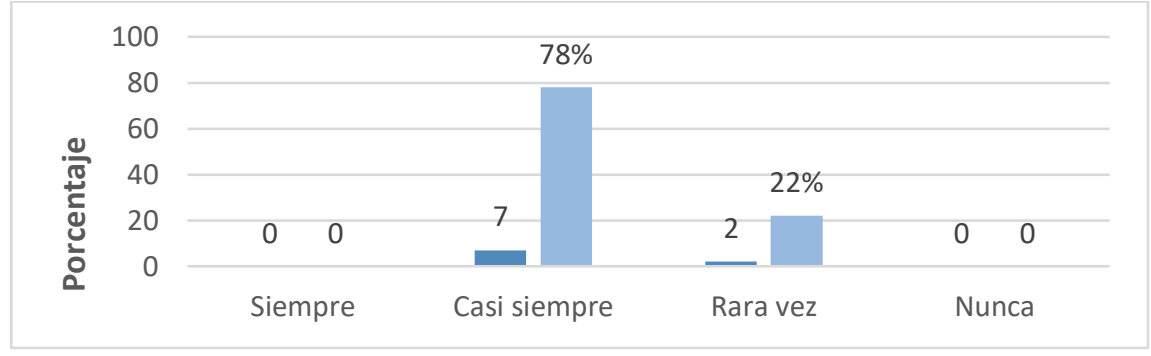

Fuente: Cuestionario aplicado a los estudiantes de bachillerato del Liceo Internacional Iberoamericano.

Los resultados generales sobre el uso de las TIC's por parte de los docentes. Nos muestra que el $78 \%$ de los encuestados casi siempre utilizan en su proceso de enseñanza, y el $22 \%$ lo utiliza rara vez. Basados en lo expuesto por Segovia Nuria (2007) existen una variedad de medios para el proceso de enseñanza - aprendizaje que deben ser utilizados en la educación. Medios que están al alcance de todos. Las autoridades de la institución deben considerar en la planificación la implementación de estrategias que conlleven al uso de medios e instrumentos tecnológicos en el desarrollo de las clases por parte de todos los docentes.

\section{Necesidades de formación docente.}

\section{Gráfico 14}

Cuestionario aplicado a docentes de bachillerato del Liceo Internacional Iberoamericano

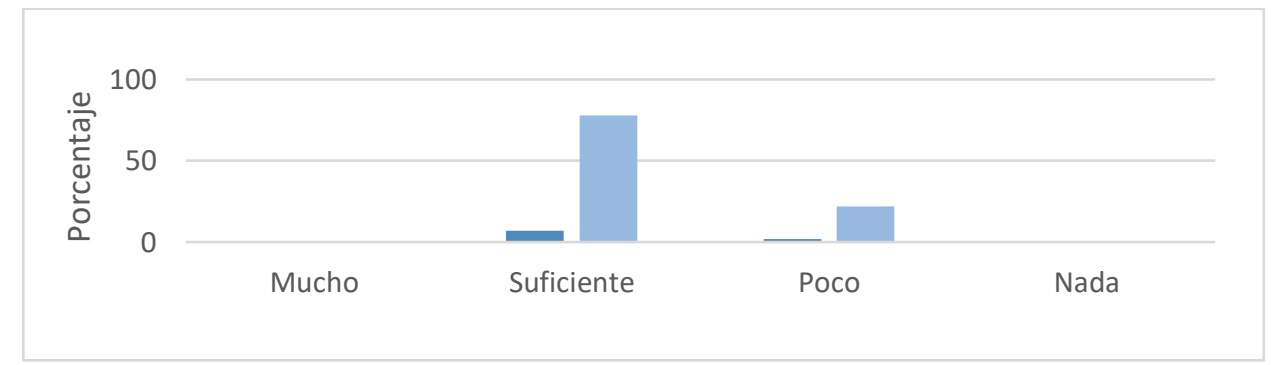

Fuente: Cuestionario aplicado a los estudiantes de bachillerato del Liceo Internacional Iberoamericano.

El 78\% de los docentes encuestados indican que conocen lo suficiente sobre los cambios en el sistema de educación a nivel de bachillerato, mientras que el $22 \%$ restante hacen referencia que sobre la temática conocen poco. Todo nuevo programa trae junto cambios del cual se debe estar debidamente informado para el direccionamiento del trabajo que se realiza día a día. Los resultados nos permiten establecer que el $22 \%$ de los docentes de la institución, requieren conocer sobre los cambios en el sistema de educación a nivel bachillerato para que el accionar diario de sus actividades este acorde a los nuevos procesos del sistema. 


\section{Gráfico 15}

Cuestionario aplicado a docentes de bachillerato del Liceo Internacional Iberoamericano

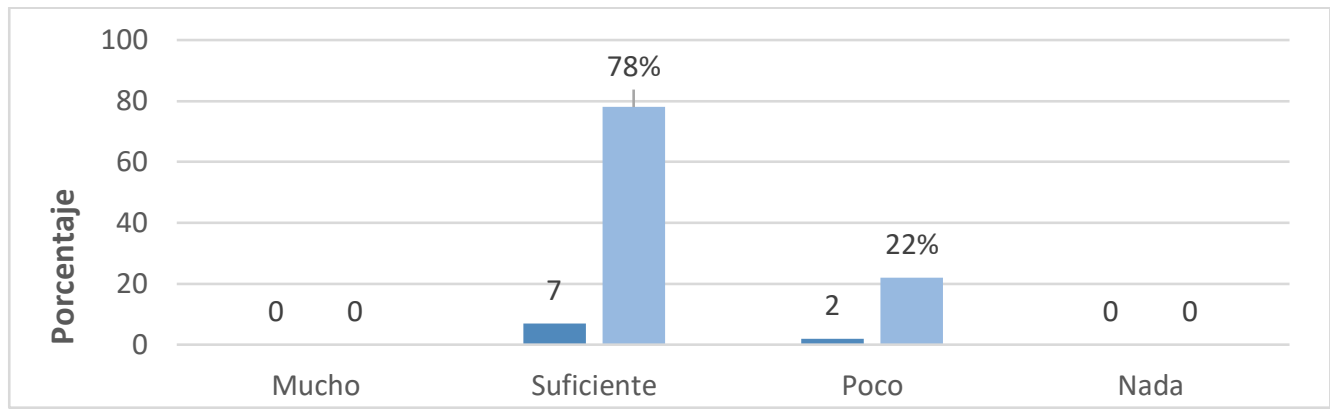

Fuente: Cuestionario aplicado a los estudiantes de bachillerato del Liceo Internacional Iberoamericano.

El $78 \%$ de los docentes manifiestan conocer lo suficiente sobre el perfil del nuevo bachiller; mientras que el 22\% de los docentes dicen no conocer. Basados en la definición del nuevo perfil del bachiller ecuatoriano por MinEduc. Consideramos que frente a la responsabilidad de los docentes con sus estudiantes y la sociedad. Todo el personal docente del nivel de bachillerato debe tener pleno conocimiento de los cambios en el sistema educativo y mantenerse informados y actualizados sobre los diferentes requerimientos para la formación de los nuevos bachilleres, en de los objetivos trazados por el MinEduc.

\section{Gráfico 16}

Cuestionario aplicado a docentes de bachillerato del Liceo Internacional Iberoamericano

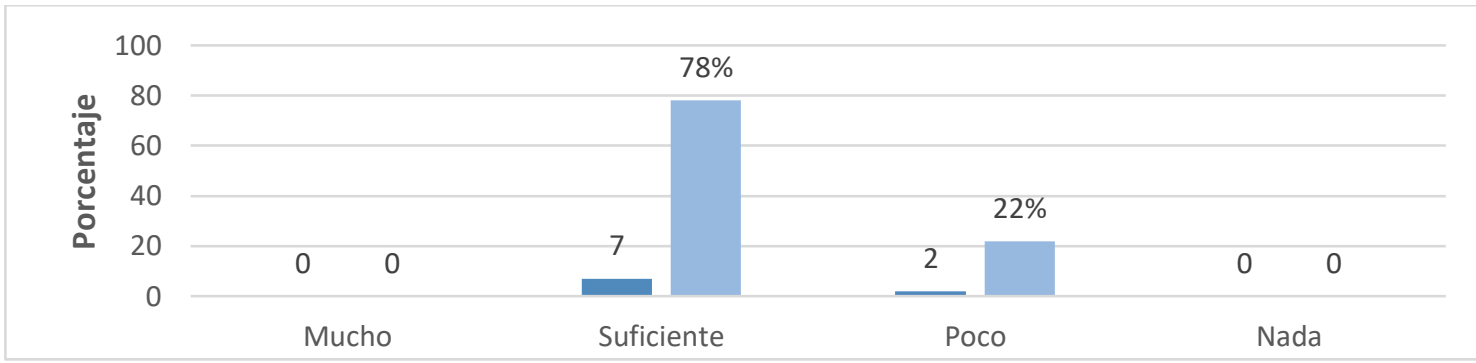

Fuente: Cuestionario aplicado a docentes de bachillerato del Liceo Internacional Iberoamericano.

Entre los 9 docentes de bachillerato, 7 docentes consideran que en el proceso de enseñanza contribuyen para el desarrollo de habilidades y destrezas de sus estudiantes. El MinEduc dice: En base a los resultados de la encuesta, se considera que todo el personal debe realizar sus actividades en concordancia a los objetivos de Ministerio de educación; las destrezas y habilidades que el estudiante logre desarrollar en su proceso de formación en la última fase como es el bachillerato, le será de gran ayuda en subida estudiantil a nivel superior y en convivencia con la sociedad. 


\section{Gráfico 17}

Cuestionario aplicado a docentes de bachillerato del Liceo Internacional Iberoamericano

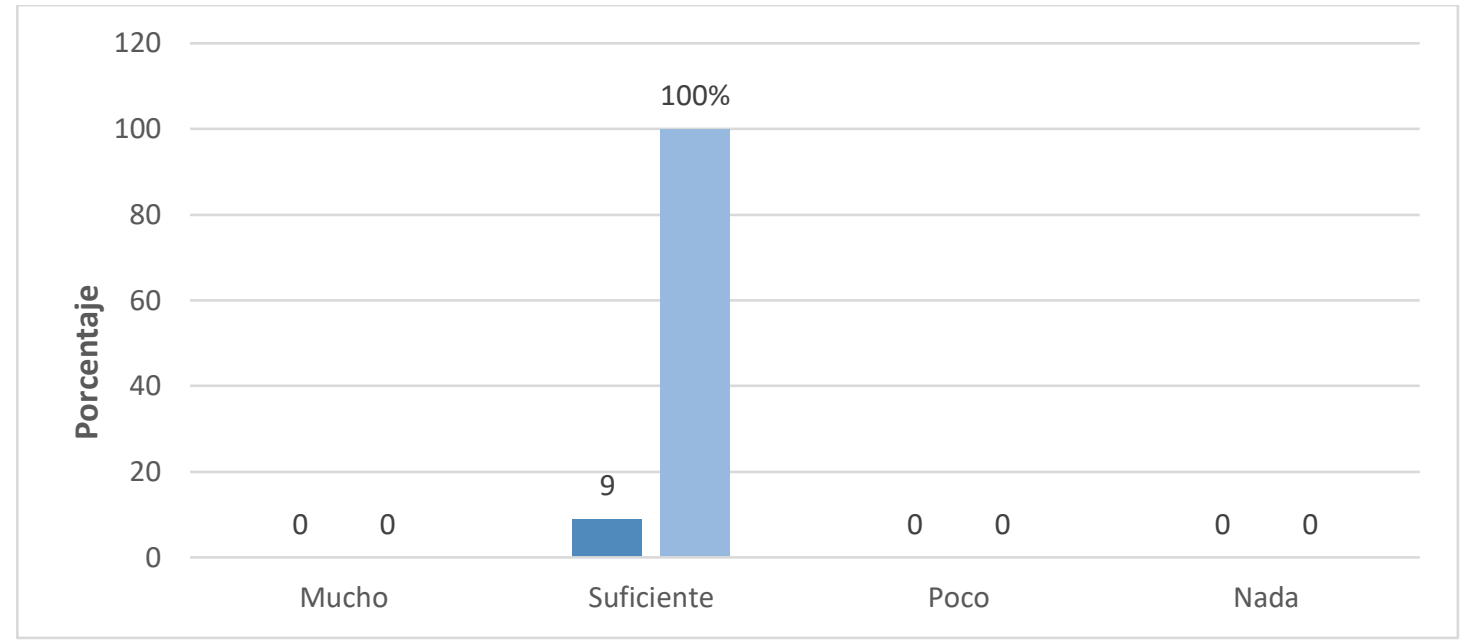

Fuente: Cuestionario aplicado a los estudiantes de bachillerato del Liceo Internacional Iberoamericano.

Nos basamos en el currículo presentado por el MinEduc específicamente en lo relacionado con el área de conocimiento interdisciplinar que comprende como asignatura para el BGU Emprendimiento y Gestión. El resultado nos permite establecer que todos los docentes se involucran en las actividades de emprendimiento y gestión de sus estudiantes. Que en otros términos también conocidos son los proyectos de vinculación con la sociedad que les permite conocer mejor la realidad del entorno.

\section{Gráfico 18}

Cuestionario aplicado a docentes de bachillerato del Liceo Internacional Iberoamericano

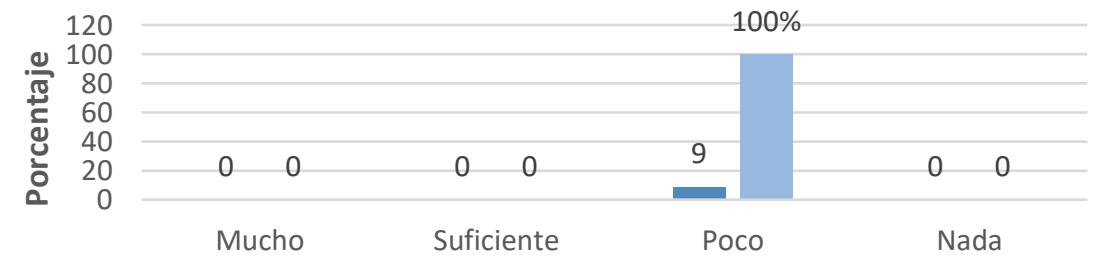

Fuente: Cuestionario aplicado a los estudiantes de bachillerato del Liceo Internacional Iberoamericano.

El 100\% de los docentes encuestados, dicen que poco participan en capacitaciones de actualización pedagógica. Basados en el criterio de Correa (2010) sobre la función del gestor educativo y de Aranéga (2013). Analizamos el resultado, podríamos estimar que no participan en capacitaciones de actualización pedagógica por mostrar desinterés en el tema o por la falta de motivación por parte de las autoridades como representantes de la institución. 


\section{Gráfico 19}

Cuestionario aplicado a docentes de bachillerato del Liceo Internacional

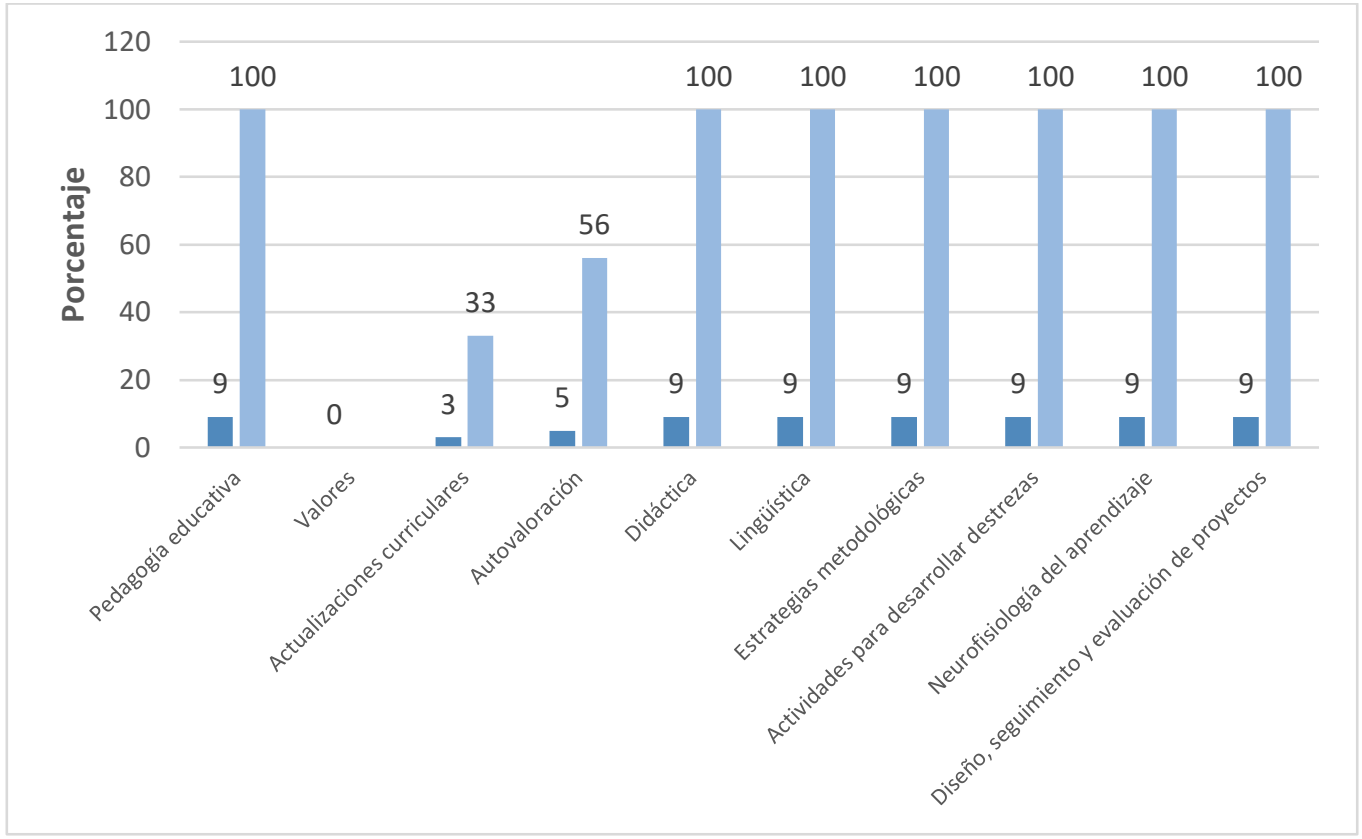

Fuente: Cuestionario aplicado a los estudiantes de bachillerato del Liceo Internacional Iberoamericano.

\section{Gráfico 20}

Cuestionario aplicado a docentes de bachillerato del Liceo Internacional Iberoamericano

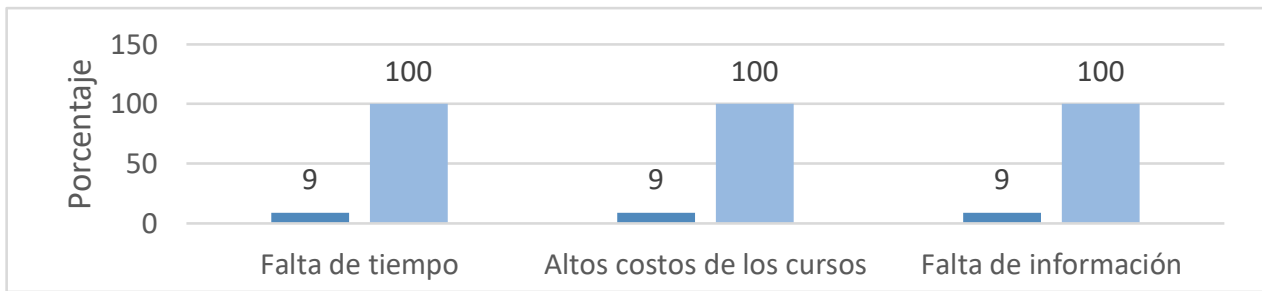

Fuente: Cuestionario aplicado a los estudiantes de bachillerato del Liceo Internacional Iberoamericano.

Se presenta en el siguiente orden: la falta de tiempo, altos costos de los cursos y la falta de información. Tomando el criterio de Liz (2017) sobre las necesidades de formación docente. Las razones que son expuestas por los docentes deben ser evaluadas.

\section{Gráfico 21}

Cuestionario aplicado a docentes de bachillerato del Liceo Internacional Iberoamericano

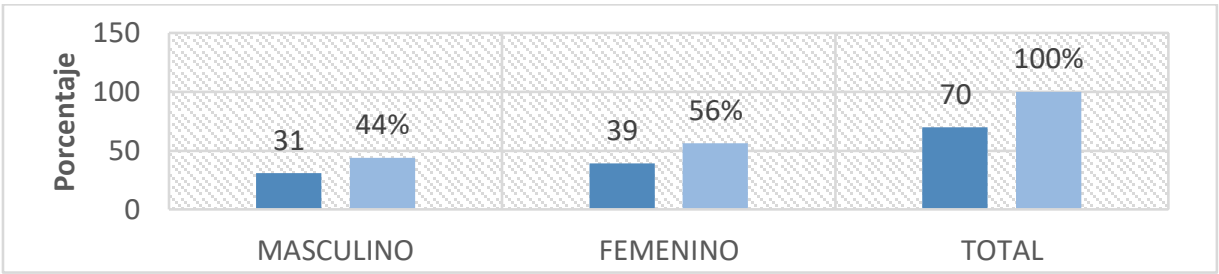

Fuente: Cuestionario aplicado a los estudiantes de bachillerato del Liceo Internacional Iberoamericano. 
De los 70 estudiantes que conforman el nivel de bachillerato, 31 estudiantes corresponden al género masculino y 39 estudiantes al género femenino. Poniendo en consideración los criterios de los modelos de análisis de necesidades de Cox y Rosett, sobre las características de los involucrados y la situación inicial en que se encuentran podemos concluir que el mayor número de los estudiantes de bachillerato son de género femenino.

\section{Grafica 22}

Cuestionario aplicado a docentes de bachillerato del Liceo Internacional Iberoamericano

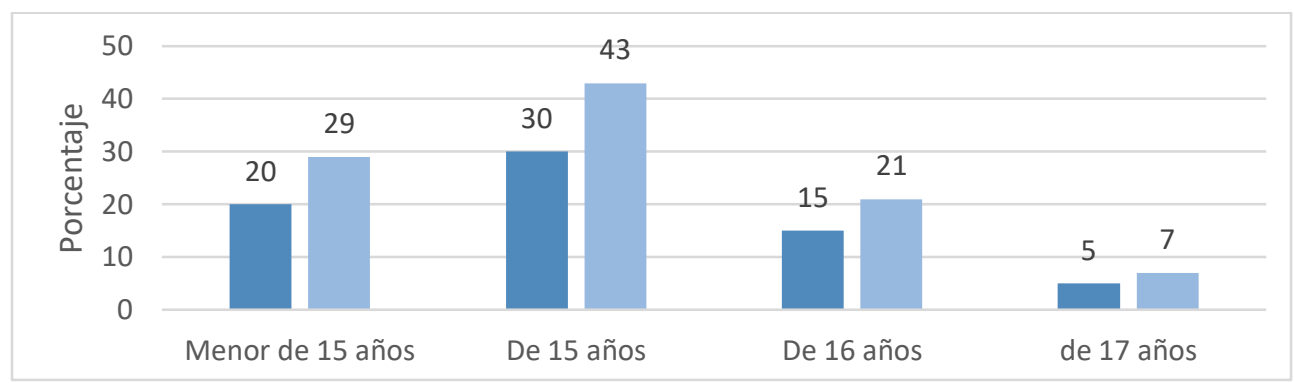

Fuente: Cuestionario aplicado a los estudiantes de bachillerato del Liceo Internacional Iberoamericano.

De la información general sobre los estudiantes se obtuvo que el $43 \%$ de los estudiantes tienen 15 años de edad, el 29\% son menores de 15 años de edad, el 21\% tienen 16 años y el 7\% tienen 17 años de edad. Para el análisis Siguiendo contemplado en los modelos de Cox y Rosett; se llega a concluir que existe el mayor porcentaje de estudiantes de bachillerato con 15 años de edad.

\section{Grafica 23}

Cuestionario aplicado a docentes de bachillerato del Liceo Internacional Iberoamericano

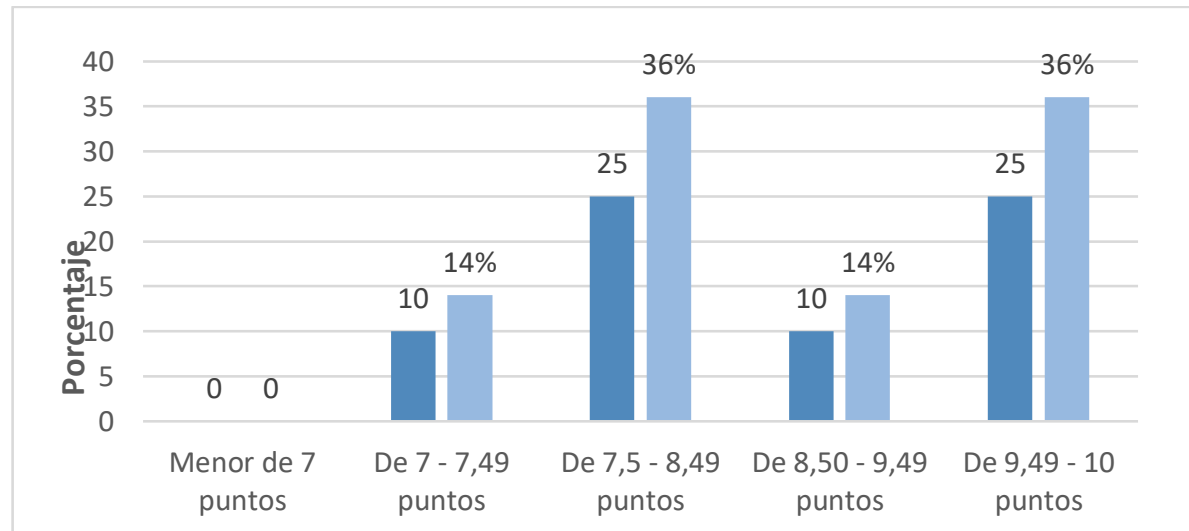

Fuente: Cuestionario aplicado a los estudiantes de bachillerato del Liceo Internacional Iberoamericano.

Según los resultados de la encuesta los promedios alcanzados en el periodo anterior por los estudiantes de bachillerato se encuentran de la siguiente manera: el $14 \%$ de un promedio entre el 7,5 - 8,49 puntos, el otro $14 \%$ de estudiantes un promedio entre el 8,50 - 9,49 puntos y el otro 36\% de estudiantes un promedio entre el 9,49 - 10 puntos. Tomando como referencia los modelos de análisis de Cox y Rosett. Concluimos que 
existe un alto porcentaje entre los promedios de 7,5-8,49 puntos y de 9,49 .10 puntos; esa es la realidad de los estudiantes de bachillerato al momento de la investigación.

\section{Grafica 24}

Cuestionario aplicado a docentes de bachillerato del Liceo Internacional Iberoamericano

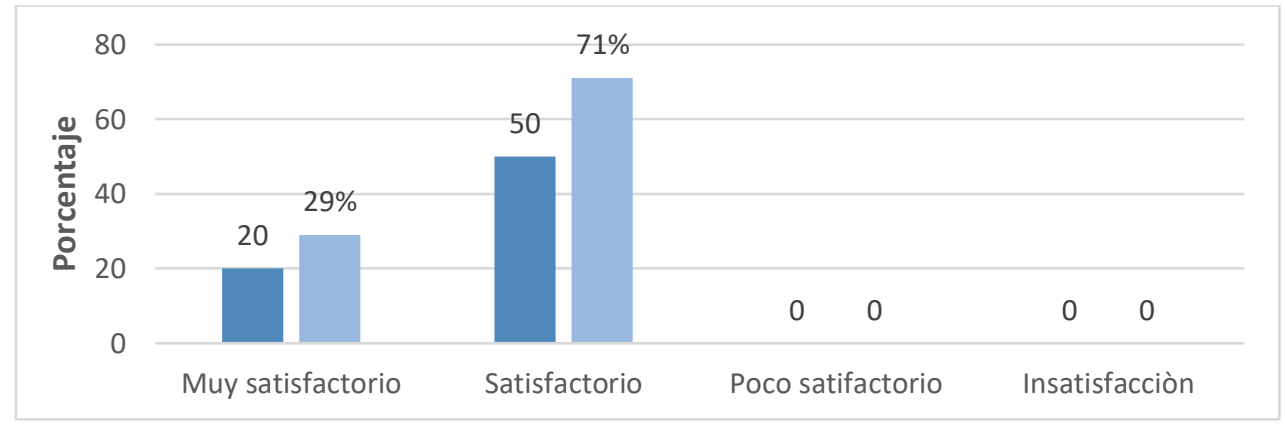

Fuente: Cuestionario aplicado a los estudiantes de bachillerato del Liceo Internacional Iberoamericano.

El $71 \%$ de los estudiantes de bachillerato consideran que el dominio de los docentes de la materia es satisfactorio, mientras que el $29 \%$ considera que es muy satisfactorio. Los modelos de Cox y Rosett han permitido conocer cuál es la situación actual del docente en relación al dominio de la materia según el criterio de los estudiantes.

\section{Gráfico 25}

Cuestionario aplicado a docentes de bachillerato del Liceo Internacional Iberoamericano

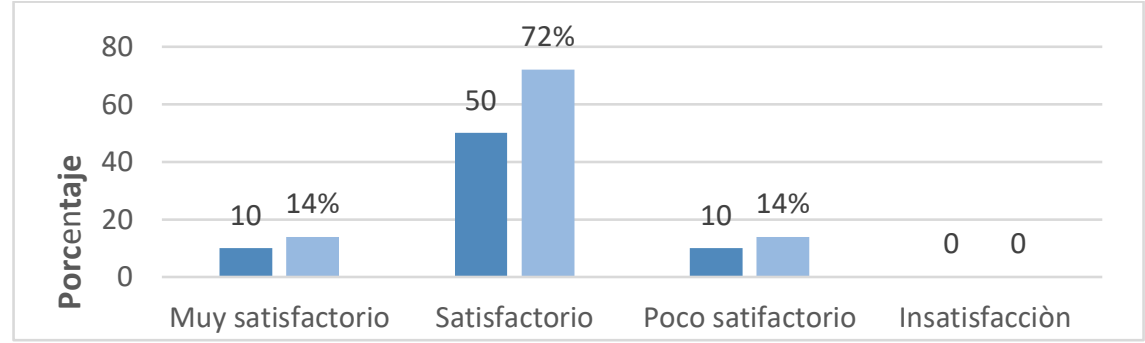

Fuente: Cuestionario aplicado a los estudiantes de bachillerato del Liceo Internacional Iberoamericano.

El 72\% de los estudiantes encuestados opinan que las estrategias de enseñanza utilizadas en clase por sus docentes son satisfactorias, un $14 \%$ de estudiantes opina que es muy satisfactorio, y un bajo porcentaje de estudiantes opina que es poco satisfactorio. El modelo de análisis de necesidades de Rosett, permite establecer que la situación actual del docente frente a los alumnos sobre las estrategias de enseñanza utilizadas en clase es satisfactoria, pero es preocupante el grupo de estudiantes que opina lo contrario, es decir existe necesidades por parte de este grupo que no son cubiertas. 


\section{Gráfico 26}

Cuestionario aplicado a docentes de bachillerato del Liceo Internacional

Iberoamericano

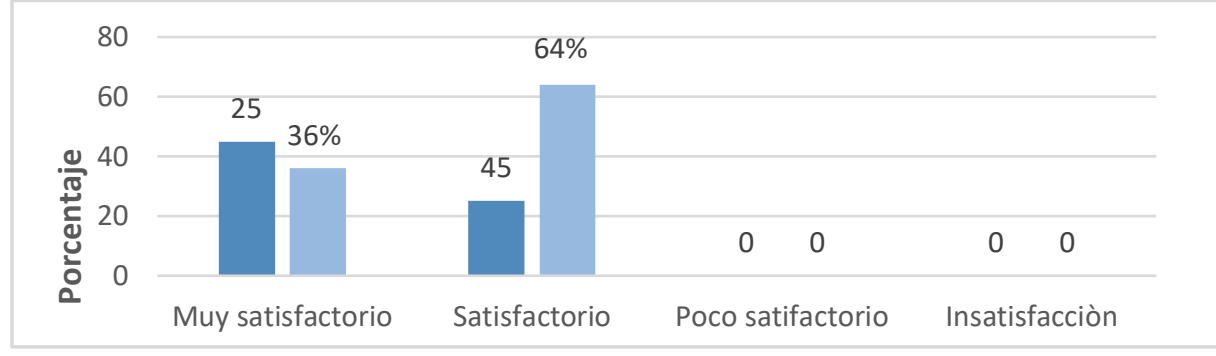

Fuente: Cuestionario aplicado a los estudiantes de bachillerato del Liceo Internacional Iberoamericano.

El 36\% de estudiantes, consideran que es satisfactorio. Esta es la situación actual del docente frente al criterio del estudiante. Se consideró el criterio del modelo de análisis de necesidades de Rosett para su análisis.

\section{Gráfico 27}

Cuestionario aplicado a docentes de bachillerato del Liceo Internacional Iberoamericano

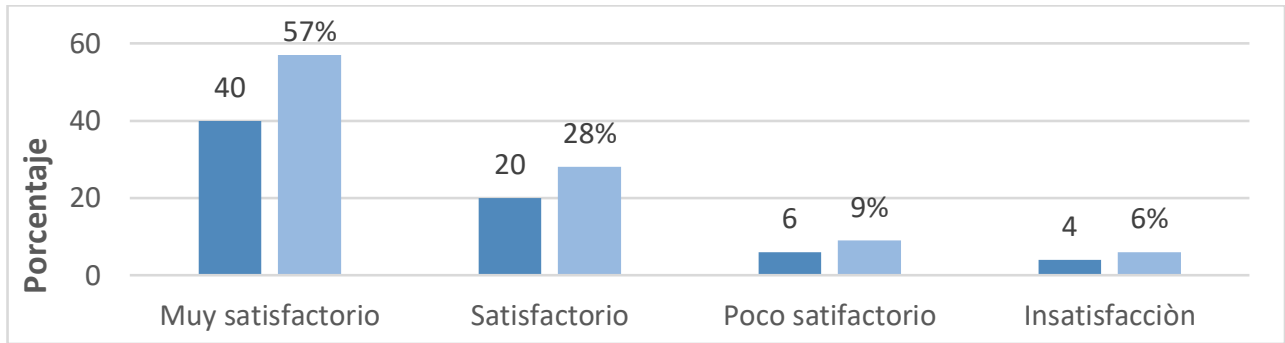

Fuente: Cuestionario aplicado a los estudiantes de bachillerato del Liceo Internacional Iberoamericano.

Las estrategias de evaluación utilizadas por los docentes; el 57\% de los encuestados opinan que son muy satisfactorio, el $28 \%$ opinan que es satisfactorio, el $9 \%$ opinan que es poco satisfactorio y el $6 \%$ se sienten insatisfechos. Siguiendo el modelo de análisis de necesidades de Rosett, se detecta la existencia de un grupo de estudiantes de bachillerato correspondiente al 15\%, que denotan la existencia de una necesidad que no ha sido cubierta por los docentes.

\section{Gráfico 28}

Cuestionario aplicado a docentes de bachillerato del Liceo Internacional Iberoamericano

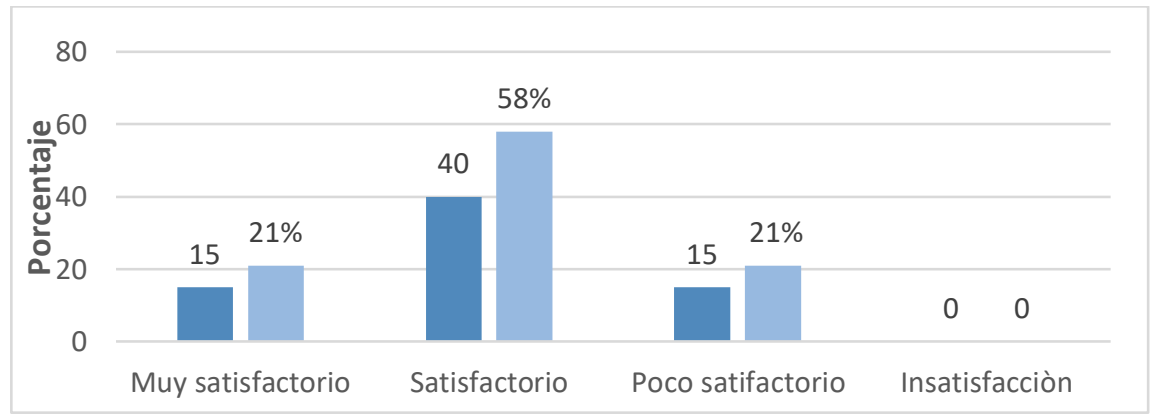

Fuente: Cuestionario aplicado a los estudiantes de bachillerato del Liceo Internacional Iberoamericano. 
Los resultados nos permiten determinar que, el $21 \%$ de estudiantes consideran que el acompañamiento académico recibido es muy satisfactorio, y el $58 \%$ opinan que es satisfactorio, y el $21 \%$ restante de los estudiantes opinan que es poco satisfactorio. Los elementos que considera en su modelo de análisis de necesidades Rosett; existe el $21 \%$ de estudiantes que se encuentran poco satisfactorio, es decir existe una necesidad insatisfecha por parte de este grupo.

\section{Satisfacción en relación a las habilidades básicas de aprendizaje que ha desarrollado.}

\section{Gráfico 29}

Cuestionario aplicado a docentes de bachillerato del Liceo Internacional Iberoamericano

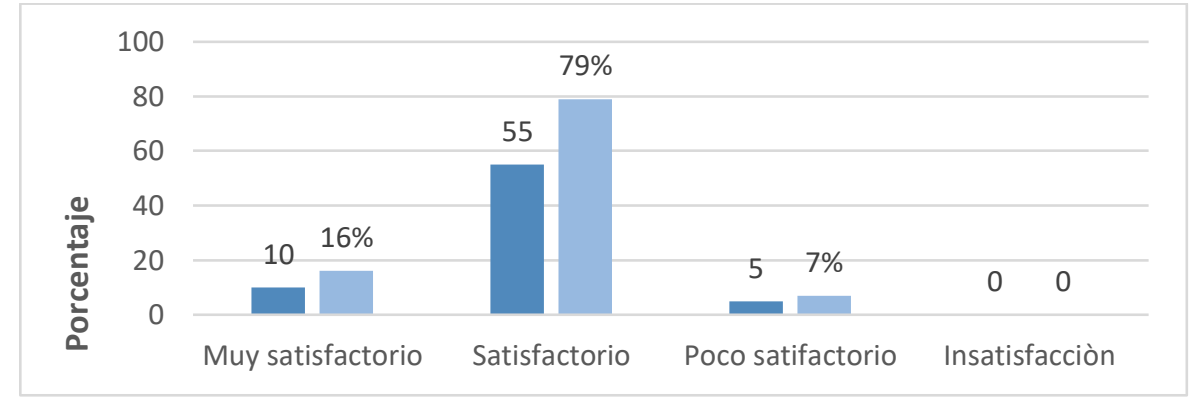

Fuente: Cuestionario aplicado a los estudiantes de bachillerato del Liceo Internacional Iberoamericano.

Las habilidades básicas desarrolladas en escritura; el 16\% de los estudiantes opinan que es muy satisfactorio, que es satisfactorio el $79 \%$, y el $7 \%$ opinan que es poco satisfactorio. Para la realización de esta pregunta nos basamos en el modelo de análisis de necesidades de Rosett y por lo establecido por el MinEduc (2016) sobre las áreas del conocimiento y asignaturas propuestas para alcanzar el perfil del nuevo bachiller. Existe un $7 \%$ de estudiantes que requieren de atención para resolver alguna falencia sentida.

\section{Gráfico 30}

Cuestionario aplicado a docentes de bachillerato del Liceo Internacional Iberoamericano

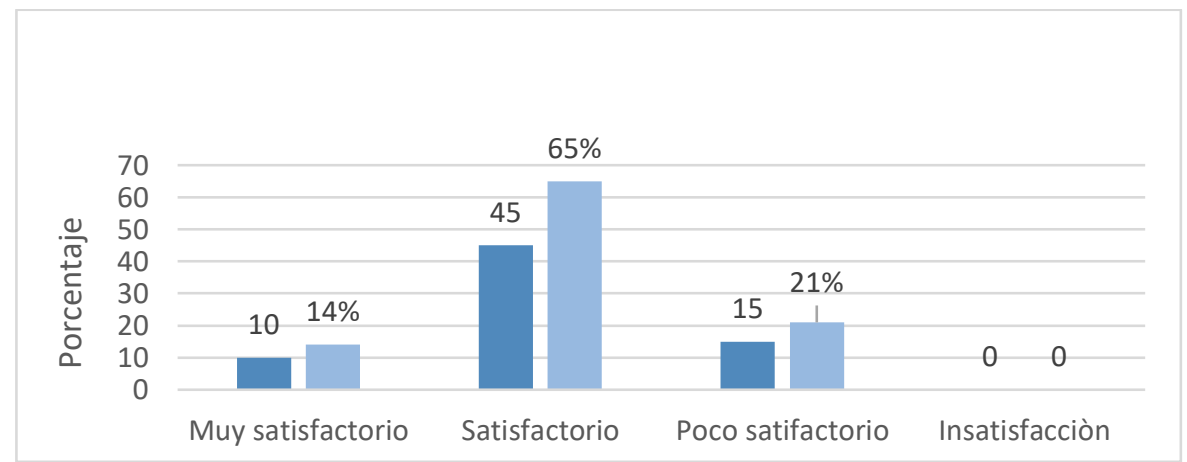

Fuente: Cuestionario aplicado a los estudiantes de bachillerato del Liceo Internacional Iberoamericano.

El resultado de la encuesta presentado en la tabla, nos muestra que el 14\% de los estudiantes opinan que la habilidad desarrollada para la lectura es muy satisfactoria, el 
$65 \%$ opinan que es satisfactorio y el $21 \%$ de los estudiantes consideran que es poco satisfactorio. Para la realización de esta pregunta nos basamos en el modelo de análisis de necesidades de Rosett y por lo establecido por el MinEduc (2016) sobre las áreas del conocimiento y asignaturas propuestas para alcanzar el perfil del nuevo bachiller. Se denota que existe un grupo de estudiantes que requieren de atención para desarrollar mejor la habilidad de lectura.

\section{Gráfico 31}

Cuestionario aplicado a docentes de bachillerato del Liceo Internacional Iberoamericano

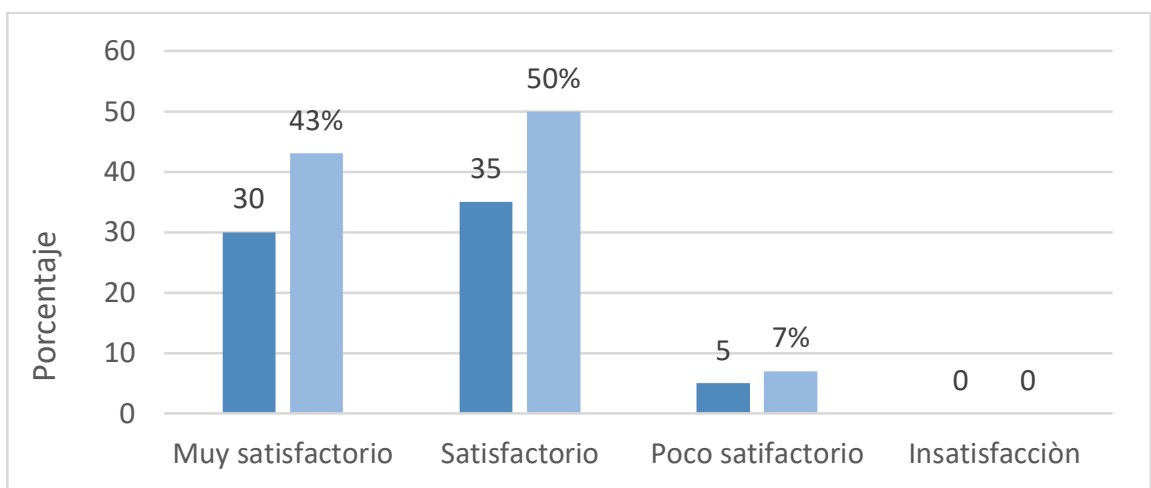

Fuente: Cuestionario aplicado a los estudiantes de bachillerato del Liceo Internacional Iberoamericano.

En relación al desarrollo de la comunicación verbal los estudiantes opinan lo siguiente: muy satisfactorio el $43 \%$ de los encuestados, para el 50\% es satisfactorio y el $7 \%$ de los estudiantes opinan que es poco satisfactorio Para la realización de esta pregunta nos basamos en el modelo de análisis de necesidades de Rosett y por lo establecido por el MinEduc (2016) sobre las áreas del conocimiento y asignaturas propuestas para alcanzar el perfil del nuevo bachiller. Como resultado final podemos establecer que existe una necesidad latente de un grupo de estudiantes que requiere su atención.

\section{Gráfico 32}

Cuestionario aplicado a docentes de bachillerato del Liceo Internacional Iberoamericano

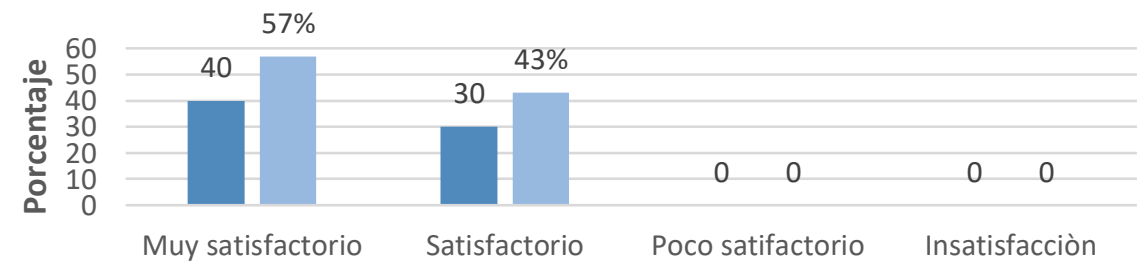

Fuente: Cuestionario aplicado a los estudiantes de bachillerato del Liceo Internacional Iberoamericano.

Para la realización de esta pregunta nos basamos en el modelo de análisis de necesidades de Rosett y por lo establecido por el MinEduc (2016) sobre las áreas del conocimiento y 
asignaturas propuestas para alcanzar el perfil del nuevo bachiller. Se podría decir que los estudiantes pueden manejar el Word y Excel sin ningún problema.

\section{Grafica 33}

Cuestionario aplicado a docentes de bachillerato del Liceo Internacional Iberoamericano

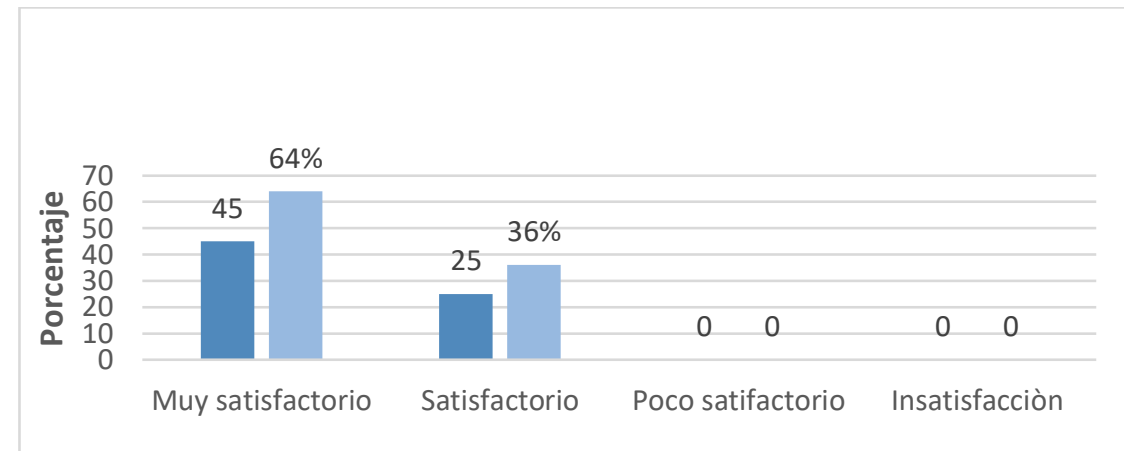

Fuente: Cuestionario aplicado a los estudiantes de bachillerato del Liceo Internacional Iberoamericano.

Con respecto a la organización de tiempo el $64 \%$ de los encuetados opinan que es muy satisfactorio y el 35\% dicen que es satisfactorio. Para la realización de esta pregunta nos basamos en el modelo de análisis de necesidades de Rosett y por lo establecido por el MinEduc (2016) sobre las áreas del conocimiento y asignaturas propuestas para alcanzar el perfil del nuevo bachiller. Podemos determinar que los estudiantes en el proceso de enseñanza, han desarrollado la habilidad con la organización de tiempo para ser puntuales y responsables en la entrega de tareas y trabajos.

\section{Grafica 34}

Cuestionario aplicado a docentes de bachillerato del Liceo Internacional Iberoamericano

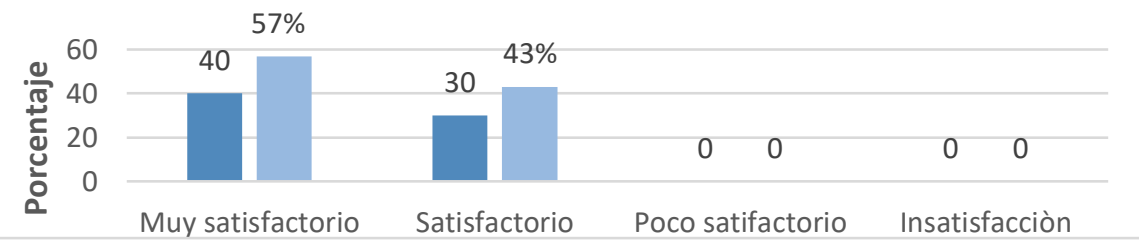

Fuente: Cuestionario aplicado a los estudiantes de bachillerato del Liceo Internacional Iberoamericano.

El resultado de la encuesta refleja que el $57 \%$ de los estudiantes de bachillerato opinan que es muy satisfactorio y el $43 \%$ opinan que es satisfactorio. Para la realización de esta pregunta nos basamos en el modelo de análisis de necesidades de Rosett y por lo establecido por el MinEduc (2016) sobre las áreas del conocimiento y asignaturas propuestas para alcanzar el perfil del nuevo bachiller. En conclusión, los estudiantes no tienen dificultades para el desarrollo de trabajos en forma individual. 


\section{Grafica 35}

Cuestionario aplicado a docentes de bachillerato del Liceo Internacional

Iberoamericano

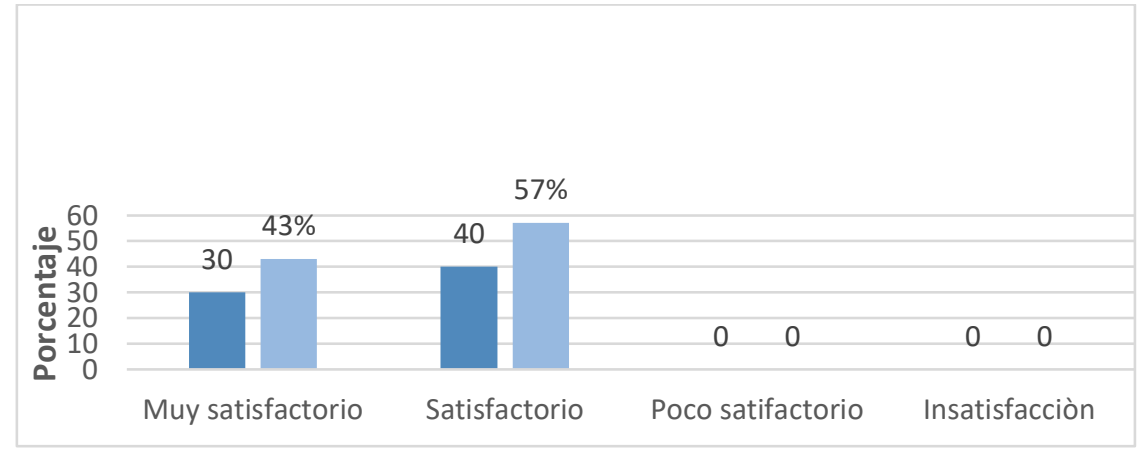

Fuente: Cuestionario aplicado a los estudiantes de bachillerato del Liceo Internacional Iberoamericano.

La encuesta nos muestra el siguiente resultado sobre la satisfacción del aprendizaje en relación al trabajo en equipo: el $43 \%$ de los encuetados consideran que es muy satisfactorio, mientras que el 57\% consideran que es satisfactorio. Para la realización de esta gpregunta nos basamos en el modelo de análisis de necesidades de Rosett y por lo establecido por el MinEduc (2016) sobre las áreas del conocimiento y asignaturas propuestas para alcanzar el perfil del nuevo bachiller. Es decir que no tienen para desarrollar trabajos en grupo.

\section{Conclusiones.}

- De acuerdo al análisis realizado los docentes de bachillerato tienen formación académica de tercer nivel; existiendo docentes que no se han formado en el ámbito de la pedagogía.

- Sobre los estudiantes del nivel de bachillerato, en relación a la enseñanza aprendizaje consideran que es muy satisfactorio y satisfactorio el ámbito en el que se están desarrollado.

- Los docentes de bachillerato de la Unidad Educativa Internacional Liceo Iberoamericano enfocan su necesidad de formación docente en diferentes campos del conocimiento tales como: pedagogía educativa; didáctica; lingüística; estrategias metodológicas; actividades para desarrollar destrezas; Neurofisiología del aprendizaje; diseño, seguimiento y evaluación de proyectos. Los obstáculos que no les ha permitido una formación continua es la falta de tiempo.

\section{Referencias bibliográficas.}

Hernandéz, R. (2010). Metodologia de la investigación. México: Mc Graw Hill.

MinEduc. (2016). BACHILLERATO GENRAL UNIFICADO. Quito: Ministerio de Educación.

Poveda, M. (2014). Necesidades de formación de los docentes de bachillerato del Colegio Gran Colombia. (Tesis de maestria), UTPL, Quito, Ecuador. 
Robles, G. (2014). Necesidades de formación de los docents de bachillerato de la Unidad Educativa Fiscomisional Pacífico Cembranos. (Tesis de maestria)., UTPL, Lago Agrio, Ecuador.

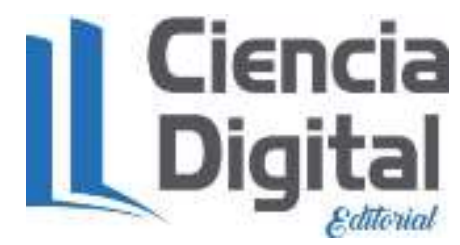




\section{PARA CITAR EL ARTÍCULO INDEXADO.}

Inca Falconí , A. F., Barahona Avecillas, M. E., Castelo Gavilanes, R. X., \& Campos Castelo, J. P. (2021). Diagnóstico de necesidades de formación de los docentes de bachillerato y su pertinencia en la enseñanza - aprendizaje en los estudiantes previo a la prueba Ser Bachiller. Unidad Educativa Internacional Ibero Americano, Cantón Riobamba, provincia de Chimb. ConcienciaDigital, 4(3.2), 95-117. https://doi.org/10.33262/concienciadigital.v4i3.2.1871

\section{UCiencia}

El artículo que se publica es de exclusiva responsabilidad de los autores y no necesariamente reflejan el pensamiento de la Revista Conciencia Digital.

El artículo queda en propiedad de la revista y, por tanto, su publicación parcial y/o total en otro medio tiene que ser autorizado por el director de la Revista Conciencia Digital.

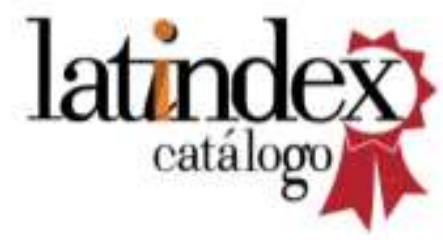

